\title{
A NOTE ON THE SPHERICAL MAXIMAL OPERATOR FOR RADIAL FUNCTIONS
}

\author{
MARK LECKBAND
}

\begin{abstract}
The spherical maximal operator for radial functions of $\mathbf{R}^{n}$ is shown to be a restricted weak type $L^{p}$ bounded operator for $p=n /(n-1)$, $n \geq 2$. The proof uses methods for restricted weak type single weight norm inequalities.
\end{abstract}

1. Introduction. The purpose of this note is to prove the endpoint norm inequality of the spherical maximal operator for radial functions.

Let $S^{n-1}=\left\{x \in \mathbf{R}^{n}:|x|=1\right\}$ and let $d \sigma$ be the normalized rotation invariant measure on $S^{n-1}$.

THEOREM. Let $f$ be a measurable radial function on $\mathbf{R}^{n}, n \geq 2$. Let $M f$ be defined by

$$
M f(x)=\sup _{t>0} \int_{S^{n-1}}|f(x-t y)| d \sigma(y) .
$$

Then we have the following inequality for $f \in L^{n /(n-1), 1}\left(\mathbf{R}^{n}\right)$ :

$$
\|M f\|_{n /(n-1), \infty} \leq C^{n}\|f\|_{n /(n-1), 1} .
$$

It is shown in [1 and 4] that the spherical maximal operator defined for functions of $\mathbf{R}^{n}, n \geq 2$, is an $L^{p}$ bounded operator for $p>n /(n-1)$. Simple examples show that this maximal operator is not a weak-type $L^{n /(n-1)}$ bounded operator on $\mathbf{R}^{n}$. However, we show that it is possible to obtain a restricted weak-type $L^{n /(n-1)}$ norm inequality for radial functions of $\mathbf{R}^{n}, n \geq 2$. This is a positive indication that the spherical maximal operator may be a restricted weak-type $L^{n /(n-1)}$ bounded operator on $\mathbf{R}^{n}, n \geq 2$. It is interesting to note that this problem also provides a nice application of restricted weak-type classes of $A p$ weights.

2. Preliminaries. The function $f$ will always be a measurable radial function of $\mathbf{R}^{n}$ for $n \geq 2$. The property of $f$ being radial will allow us to interpret $M f$ as the one-dimensional maximal operator $M_{n} f$ on $(0, \infty)$ defined below.

DEFINITION 1. Let $s>0$ and $n \geq 2$. We let $M_{n} f$ denote the maximal operator defined by

$$
M_{n} f(s)=\sup _{r>0} \int \phi_{n, I_{r, s}}(t)|f(t)| t^{n-1} d t
$$

where $I_{r, s}=[|s-r|, s+r]=[a, b] \subset(0, \infty)$, and

$$
\phi_{n, I_{r, s}}(t)=\frac{4 \Gamma(n / 2)}{\Gamma((n-1) / 2) \Gamma(1 / 2)}\left[\frac{2\left(b^{2}-t^{2}\right)^{1 / 2}\left(t^{2}-a^{2}\right)^{1 / 2}}{\left(b^{2}-a^{2}\right)}\right]^{n-3} \frac{t^{2-n}}{\left(b^{2}-a^{2}\right)} \chi_{I_{r, s}}(t) .
$$

Received by the editors February 24, 1986.

1980 Mathematics Subject Classification (1985 Revision). Primary 42B25. 
We shall need to consider other maximal operators which we will now define.

$$
M_{n, 0} f(s)=\sup \int \phi_{n, I_{r, s^{\prime}}}(t)|f(t)| t^{n-1} d t,
$$

where the sup is taken over all $r, s^{\prime}>0$ such that $0<r<s^{\prime}$ and $\left|s-s^{\prime}\right|<r / 2$.

$$
M_{n, k} f(s)=\sup _{2^{k-1} s<r<2^{k}} \int \phi_{n, I_{r, s}}(t)|f(t)| t^{n-1} d t
$$

for $k=1,2,3, \ldots$

It is to be observed that the maximal operator $M_{n}$ is comprised of $M_{n, 0}$ and $M_{n, k}$; in particular, $M_{n} f \leq \sum_{j=0}^{\infty} M_{n, j} f$. We use $I_{r, s^{\prime}}$ instead of $I_{r, s}$ in defining $M_{n, 0} f$ in order to control $M_{n, k} f, k \geq 1$, by $M_{n, 0} f$. This shall be seen in the proof of statement (b) of Theorem 1.

LEMMA 1. Let $x \in \mathbf{R}^{n}, n \geq 2$, and $x \neq 0$. Then

$$
M f(x)=M_{n} f(|x|) .
$$

PROOF. We may assume $f \geq 0$. Let $R_{x}=\{t x: 0 \leq t \leq 1\}$ be the line segment from 0 to $x$, and let $S^{n-1}(x, r)=\left\{y \in \mathbf{R}^{n}:|x-y|=r\right\}$. For $z \in S^{n-1}(x, r)$, let $\psi(z)$ be the angle with vertex at $x$ measured from $R_{x}$ to the line segment formed by $z$ and $x$. Then

$$
\int f(x-r y) d \sigma(y)=\omega_{n} \int_{0}^{\pi} f(\rho) \sin ^{n-2} \psi d \psi
$$

where $\omega_{n}=\Gamma(n / 2) / \Gamma((n-1) / 2) \Gamma(1 / 2)$ and $\rho^{2}=r^{2}+|x|^{2}-2 r|x| \cos (\psi)$. To see this, we first observe that the integrand $f$ depends only on $\psi$. Holding $\psi$ constant and integrating out the other $n-2$ variables gives the surface area of the $n-2$ dimensional sphere of radius $\sin \psi$. The $n-2$ dimensional surface area is $\left[(n-1) \pi^{(n-1) / 2} / \Gamma((n+1) / 2)\right] \sin ^{n-2} \psi$. We may find the normalizing constant by using the well-known formula

$$
\int_{0}^{\pi} \sin ^{n-2} \psi d \psi=\frac{\Gamma((n-1) / 2) \Gamma(1 / 2)}{\Gamma(n / 2)} .
$$

By a change of variables and using the formulas

(i) $\rho d \rho=|x| r \sin \psi d \psi$,

(ii) $[\sin \psi]^{n-3}=\left[\left|(|x|+r)^{2}-\rho^{2}\right|^{1 / 2}\left|\left(|x|^{2}-r^{2}\right)-\rho^{2}\right|^{1 / 2} / 2|x| r\right]^{n-3}$, we derive

$$
\omega_{n} \int_{0}^{\pi} f(\rho) \sin ^{n-2} \psi d \psi=\int \phi_{n, I}(t) f(t) t^{n-1} d t
$$

where $I=[a, b]=[|| x|-r|,|x|+r] \subset(0, \infty)$, and

$$
\phi_{n, I}(t)=4 \omega_{n}\left[\frac{2\left(b^{2}-t^{2}\right)^{1 / 2}\left(t^{2}-a^{2}\right)^{1 / 2}}{\left(b^{2}-a^{2}\right)}\right]^{n-3} \frac{t^{2-n}}{\left(b^{2}-a^{2}\right)} \cdot \chi_{I}(t) .
$$

This completes the proof of Lemma 1 . 
3. The maximal operator $M_{n}$ behaves badly when the test intervals $I_{r, s}$, used in evaluating $M_{n}$, have $r>s$. To control $M_{n}$, we decompose this operator into $M_{n, 0}+\sum_{j=1}^{\infty} M_{n, j}$ as mentioned in Definition 1 . The operator $M_{n, 0}$ is the good maximal operator that ultimately controls $M_{n, j}$ and thereby $M_{n}$.

Let $\nu_{n}$ be the measure on $(0, \infty)$ defined by $\nu_{n}(E)=\int_{E} t^{n-1} d t$ for measurable $E \subset(0, \infty)$. Then $M_{n, 0} f(s)=\sup \int \phi_{n, I}(t) f(t) d \nu_{n}(t)$, where the sup is taken over all $\phi_{n, I}, I$ is contained in $(0, \infty)$ whose center $s^{\prime}$ satisfies $\left|s-s^{\prime}\right|<\frac{1}{4}|I|$. We shall show in Theorem 1(a) that $M_{n, 0}$ maps $L_{\nu_{n}}^{n /(n-1), 1}$ boundedly into $L_{\nu_{n}}^{n /(n-1), \infty}$. To do this we shall use the methods of [2] which are particularly suitable for restricted weak-type norm inequalities. To begin, we denote $g_{\nu_{n}}^{*}$ to be the nonincreasing rearrangement of $g$ with respect to $\nu_{n}$, that is $g_{\nu_{n}}^{*}(t)=\inf \left\{s: \nu_{n}\{|g|>s\} \leq t\right\}$. Then we have the following lemma.

LEMMA 2. $\left(M_{n, 0} f\right)_{\nu_{n}}^{*}(\xi) \leq A \int_{0}^{\infty} \Phi_{n}(t) f_{\nu_{n}}^{*}(t \xi) d t$, where $0<\xi<\infty$, and

$$
\Phi_{n}(t)=\sup _{I \subset(0, \infty)} \nu_{n}(I)\left(\phi_{n, I}\right)_{\nu_{n}}^{*}\left(\nu_{n}(I) t\right) .
$$

ProOF. We observe that Theorem 1 of [2] only requires the Besicovitch covering lemma. Even though the Besicovitch lemma is unnecessary for intervals, we have defined $M_{n, 0} f$ so that the proof of Theorem 1 of [2] implies our lemma.

From the above rearrangement inequality one easily sees that if $\Phi_{n} \in L^{n, \infty}(0, \infty)$, then $\left\|M_{n, 0} f\right\|_{n /(n-1), \infty, \nu_{n}} \leq A\|f\|_{n /(n-1), 1, \nu_{n}}$.

LEMMA 3. We have $\Phi_{n} \in L^{n, \infty}(0, \infty)$ for $n \geq 2$.

Proof. Let $I=[a, b] \subset(0, \infty)$. Then $\left(\phi_{n, I}\right)_{\nu_{n}}^{*}\left(\nu_{n}(I) t\right)=0$ for $t \geq 1$ since the support of $\phi_{n, I}$ is contained in $I$. Thus we only need to show for $0<t<1$ that

$$
L_{n, I}(t)=\nu_{n}(I)\left(\phi_{n, I}\right)_{\nu_{n}}^{*}\left(\nu_{n}(I) t\right) \leq c t^{-1 / n} \text {. }
$$

To do this we first restrict ourselves to $n \geq 3$. We consider the two cases $a>b / 2>0$ and $0<a \leq b / 2$.

Let $a>b / 2>0$ and $n \geq 3$. Then $s^{n-2} \geq s \cdot a^{n-3} \geq 2^{3-n} s \cdot b^{n-3}$ and $\left(b^{2}-s^{2}\right)^{1 / 2}\left(s^{2}-a^{2}\right)^{1 / 2} \leq b^{2}-a^{2} \leq 2 b(b-a)$. Thus we observe

$$
\begin{aligned}
\phi_{n, I}(s) & =\frac{4 \omega_{n} s^{2-n}}{b^{2}-a^{2}}\left|\frac{2\left(b^{2}-s^{2}\right)^{1 / 2}\left(s^{2}-a^{2}\right)^{1 / 2}}{\left(b^{2}-a^{2}\right)}\right|^{n-3} \chi_{I}(s) \\
& \leq \frac{4^{n-2} \omega_{n}}{b^{n-2}(b-a)} \frac{\chi_{I}(s)}{s}
\end{aligned}
$$

and so

$$
\left(\phi_{n, I}\right)_{\nu_{n}}^{*}\left(\nu_{n}(I) t\right) \leq \frac{4^{n-2} \omega_{n}}{b^{n-2}(b-a)}\left(\frac{\chi_{I}}{s}\right)_{\nu_{n}}^{*}\left(\nu_{n}(I) t\right) .
$$

To compute the rearrangement on the right, we equate $\nu_{n}\{s \in I: 1 / s>\tau\}$ with $\nu_{n}(I) t=\left(b^{n}-a^{n}\right) t / n$, or $\left[(1 / \tau)^{n}-a^{n}\right]=\left(b^{n}-a^{n}\right) t$. Solving for $\tau$ we find

$$
\left(\chi_{I} / s\right)_{\nu_{n}}^{*}\left(\nu_{n}(I) t\right)=\left[\left(b^{n}-a^{n}\right) t+a^{n}\right]^{-1 / n} .
$$

Finally $\nu_{n}(I) \leq(b-a) b^{n-1}$ and the above implies (for $a>b / 2>0$ ),

$$
L_{n, I}(t) \leq 4^{n-2} \omega_{n}\left(\frac{b^{n}}{\left(b^{n}-a^{n}\right) t+a^{n}}\right)^{1 / n} \leq c_{n} \omega_{n} t^{-1 / n} .
$$


Now we consider $0<a<b / 2$ and $n \geq 3$. Given $I=[a, b]$ and $I_{0}=[0, b]$, then $\frac{7}{8} \nu_{n}\left(I_{0}\right) \leq \nu_{n}(I) \leq \nu_{n}\left(I_{0}\right)$. Moreover we also have

$$
\left(b^{2}-s^{2}\right)^{1 / 2}\left(s^{2}-a^{2}\right)^{1 / 2} /\left(b^{2}-a^{2}\right) \leq s /\left(b^{2}-a^{2}\right)^{1 / 2} \leq \frac{4}{3} \cdot s / b .
$$

This implies

$$
\phi_{n, I}(s) \leq \frac{4^{2} \omega_{n} 2^{n-3}}{3 b^{2} b^{n-3}}\left(\frac{4}{3}\right)^{n-3}\left(\frac{\chi_{I_{0}}(s)}{s}\right)
$$

or

$$
\left(\phi_{n, I}\right)_{\nu_{n}}^{*}\left(\nu_{n}(I) t\right) \leq \frac{2^{2 n-7} \omega_{n}}{3^{n-2}}\left(\frac{\chi_{I_{0}}}{s}\right)_{\nu_{n}}^{*}\left(\frac{7}{8} \nu_{n}\left(I_{0}\right) t\right)
$$

If we let $t^{\prime}=\left(\frac{7}{8}\right) t$, then the computation of the rearrangement on the right is exactly the same as before. Hence for $0<a<b / 2$ we derive $L_{n, I}(t) \leq c_{n} \omega_{n} t^{-1 / n}$. This completes the proof for $n \geq 3$.

For the case $n=2$, we begin with

$$
\begin{aligned}
\phi_{2, I}(s) & =2 \omega_{2}\left[\left(b^{2}-s^{2}\right)^{-1 / 2}\left(s^{2}-a^{2}\right)^{-1 / 2}\right] \chi_{I}(s) \\
& \leq \frac{c}{\left(b^{2}-a^{2}\right)^{1 / 2}}\left[\left(b^{2}-s^{2}\right)^{-1 / 2}+\left(s^{2}-a^{2}\right)^{-1 / 2}\right] \chi_{I}(s) .
\end{aligned}
$$

Thus

$$
\begin{aligned}
\left(\phi_{2, I}\right)_{\nu_{2}}^{*}\left(\nu_{2}(I) t\right) \leq \frac{c}{\left(b^{2}-a^{2}\right)^{1 / 2}}\left[\left(\frac{\chi_{I}}{\left(b^{2}-s^{2}\right)^{1 / 2}}\right)_{\nu_{2}}^{*}\left(\frac{\nu_{2}(I) t}{2}\right)\right. & \\
& \left.+\left(\frac{\chi_{I}}{\left(s^{2}-a^{2}\right)^{1 / 2}}\right)_{\nu_{2}}^{*}\left(\frac{\nu_{2}(I) t}{2}\right)\right] .
\end{aligned}
$$

To compute the first rearrangement we must find $\tau$ that satisfies

$$
\nu_{2}\left\{s \in I:\left(b^{2}-s^{2}\right)^{-1 / 2}>\tau\right\} \quad \text { equals } \quad \nu_{2}(I) t / 2=\left(b^{2}-a^{2}\right) t / 4 .
$$

We find $\tau=2 /\left[\left(b^{2}-a^{2}\right)^{1 / 2} \cdot t^{1 / 2}\right]$. Similarly for the second rearrangement, we must find $\tau$ that satisfies $\nu_{2}\left\{s \in I:\left(s^{2}-a^{2}\right)^{1 / 2}>\tau\right\}$ equals $\nu_{2}(I) t / 2$. Again we find that $\tau=2 /\left[\left(b^{2}-a^{2}\right)^{1 / 2} \cdot t^{1 / 2}\right]$. Thus we obtain

$$
L_{2, I}(t) \leq c t^{-1 / 2}
$$

and this completes the proof.

We are now able to demonstrate that $M_{n, 0}$ is restricted weak-type $p=n /(n-1)$ for functions of $(0, \infty)$. In the next theorem we shall also prove that $M_{n, k}$ is also restricted weak-type $p=n /(n-1)$ with a norm constant that rapidly converges to 0 as $k \rightarrow \infty$. This is done by relating in some sense $M_{n, k}$ to $M_{n, 0}$.

THEOREM 1. Suppose $f \in L_{\nu_{n}}^{n /(n-1), 1}, n \geq 2$. Then

$$
\left\|M_{n, 0} f\right\|_{n /(n-1), \infty, \nu_{n}} \leq C_{n}\|f\|_{n /(n-1), 1, \nu_{n}},
$$

$$
\left\|M_{n, k} f\right\|_{n /(n-1), \infty, \nu_{n}} \leq C_{n}\left(\frac{1}{2^{(k-1)(n-1)}}\right)^{(n-1) / n}\|f\|_{n /(n-1), 1, \nu_{n}}
$$

for $k=1,2,3, \ldots$. 
PROOF. To prove (a) we begin with

$$
\begin{aligned}
\left\|M_{n, 0} f\right\|_{n /(n-1), \infty, \nu_{n}} & =\sup _{\alpha>0} \alpha\left[\nu_{n}\left\{M_{n, 0} f>\alpha\right\}\right]^{(n-1) / n} \\
& =\sup _{\xi>0} \xi^{(n-1) / n} \cdot\left(M_{n, 0} f\right)_{\nu_{n}}^{*}(\xi) .
\end{aligned}
$$

The last equality is due to the fact that the decreasing rearrangement is the inverse function of the distribution function. Applying Hölder's inequality to the right side of Lemma 2, we obtain

$$
\left(M_{n, 0} f\right)_{\nu_{n}}^{*}(\xi) \leq A\|\Phi\|_{n, \infty}\left\|f_{\nu_{n}}^{*}(\cdot \xi)\right\|_{n /(n-1), 1} .
$$

By Lemma 3, $\|\Phi\|_{n, \infty} \leq C_{n}$. Using

$$
\left\|f_{\nu_{n}}^{*}(\cdot \xi)\right\|_{n /(n-1), 1}=\xi^{-n /(n-1)}\|f\|_{n /(n-1), 1, \nu_{n}}
$$

and the above completes the proof of (a).

To prove (b), consider a $k \geq 1$ and fix $\alpha>0$. Let $E=\left\{s \in(0, \infty): M_{n, k} f(s)>\right.$ $\alpha\}$. Given $s \in E$, then $\int \phi_{n, I_{r(s), s}}(t)|f(t)| t^{n-1} d t>\alpha$ for some $r(s)$ with $2^{(k-1)} s \leq$ $r(s) \leq 2^{k} s$. Let $J_{s}=(0, s]$ and $J_{s, r(s)}=J_{s}+r(s)$. We have $E \subset \bigcup_{s \in E} J_{s}$. Since $\left\{J_{t}\right\}$ is an increasing sequence of intervals, we also have $E \subset J_{\tau}$ where $\tau=$ $\sup \left\{s: M_{n, k} f(s)>\alpha\right\}$.

We observe that $M_{n, 0} f(r(\tau))>\alpha / 2$ for $r(\tau) \in \bar{J}_{\tau, r(\tau)}=[r(\tau), \tau+r(\tau)]$. Thus $M_{n, 0} f(u)>\alpha / 2$ for $u \in \frac{1}{2} I_{r(\tau), \tau}=\left[r_{\tau}-\tau / 2, r_{\tau}-\tau / 2\right]$. This implies

$$
\nu_{n}\left(J_{\tau, r(\tau)}\right) \leq C_{n} \nu_{n}\left(\frac{1}{2} I_{r(\tau), \tau}\right) \leq C \nu_{n}\left\{M_{n, 0} f>\alpha / 2\right\}
$$

We compute $\nu_{n}\left(J_{\tau}\right)=\tau^{n} / n$ and

$$
\nu_{n}\left(J_{\tau, r(\tau)}\right) \geq \nu_{n}\left[2^{k-1} \tau,\left(2^{k-1}+1\right) \tau\right] \geq 2^{(k-1)(n-1)} \tau^{n} .
$$

Hence

$$
\begin{aligned}
\nu_{n}(E) & \leq\left(\frac{1}{2^{(k-1)(n-1)}}\right) \nu_{n}\left(J_{\tau, r(\tau)}\right) \\
& \leq C_{n}\left(\frac{1}{2^{(k-1)(n-1)}}\right) \nu_{n}\left\{M_{n, 0} f>\frac{\alpha}{2}\right\} .
\end{aligned}
$$

Now using (a) we obtain (b) to complete the proof.

It is interesting to note that when $n=3, M_{n, 0} f$ can be related to the HardyLittlewood maximal operator applied to $f\left(u^{1 / 2}\right)$ by letting $u^{2}=t$. Also, it is easily seen that the weight $w(u)=u^{1 / 2} \in A p$ for $p>3 / 2$ on $(0, \infty)$ [3]. Part (a) of Theorem 1 implies that $u^{1 / 2}$ belongs to what could be called restricted weak-type $A p$ for $p=3 / 2$.

We come to the main result of this paper.

THEOREM 2. Let $f$ be a radial function that belongs to $L^{n /(n-1), 1}\left(\mathbf{R}^{n}\right), n \geq 2$. Then

$$
\|M f\|_{n /(n-1), \infty} \leq C_{n}\|f\|_{n /(n-1), 1} .
$$

ProOF. Fix $\alpha>0$. Using Lemma 1, we have $\left|\left\{x \in \mathbf{R}^{n}: M f(x)>\alpha\right\}\right|=$ $\sigma_{n} \nu_{n}\left\{s \in(0, \infty): M_{n} f(s)>\alpha\right\}$, where $\sigma_{n}=\left|S^{n-1}\right|_{n-1}$. By Definition $1, M_{n} f(s)$ $\leq M_{n, 0} f(s)+\sum_{k=1}^{\infty} M_{n, k} f(s)$. Thus, since $\|\cdot\|_{n /(n-1), \infty, \nu_{n}}$ is equivalent to a norm, 
we obtain

$$
\left\|M_{n} f\right\|_{n /(n-1), \infty, \nu_{n}} \leq C \sum_{k=0}^{\infty}\left\|M_{n, k} f\right\|_{n /(n-1), \infty, \nu_{n}} \leq C_{n}\|f\|_{n /(n-1), 1, \nu_{n}},
$$

where the last inequality was obtained by Theorem 1(a) and (b). Finally we observe that $\|f\|_{n /(n-1), 1, \nu_{n}}=B_{n}\|f\|_{n /(n-1), 1}$ to complete the proof.

\section{REFERENCES}

1. J. Bourgain, Averages in the plane over convex curves and maximal operators, preprint.

2. M. Leckband and C. Neugebauer, A general maximal operator and the $A_{p}$-condition, Trans. Amer. Math. Soc. 275 (1983), 821-831.

3. B. Muckenhoupt, Weighted norm inequalities for the Hardy maximal function, Trans. Amer. Math. Soc. 165 (1972), 207-226.

4. E. Stein and S. Wainger, Problems in harmonic analysis related to curvature, Bull. Amer. Math. Soc. 84 (1978), 1239-1295.

Department of Mathematical SCIENCES, Florida International UniVersity, MIAMI, FLORIDA 33199 